Article

\title{
Green Synthesis of Ag-Au Bimetallic Nanocomposites Using Waste Tea Leaves Extract for Degradation Congo Red and 4-Nitrophenol
}

\author{
Chun-Won Kang * and Haradhan Kolya *
}

Citation: Kang, C.-W.; Kolya, H. Green Synthesis of Ag-Au Bimetallic Nanocomposites Using Waste Tea Leaves Extract for Degradation Congo Red and 4-Nitrophenol. Sustainability 2021, 13, 3318. https://doi.org/10.3390/su13063318

Academic Editor: Andreas N. Angelakis

Received: 25 February 2021

Accepted: 15 March 2021

Published: 17 March 2021

Publisher's Note: MDPI stays neutral with regard to jurisdictional claims in published maps and institutional affiliations.

Copyright: (c) 2021 by the authors. Licensee MDPI, Basel, Switzerland. This article is an open access article distributed under the terms and conditions of the Creative Commons Attribution (CC BY) license (https:// creativecommons.org/licenses/by/ $4.0 /)$.
Department of Housing Environmental Design and Research Institute of Human Ecology, College of Human Ecology, Jeonbuk National University, Jeonju 54896, Korea

* Correspondence: kcwon@jbnu.ac.kr (C.-W.K.); haradhankoley@gmail.com (H.K.)

\begin{abstract}
A sustainable supply of pure water is a great challenge in most developing and thirdworld countries. Nanomaterial-based technology offers technological development for wastewater purification. Nanocatalysis hydrogenation of nitroarene and dye molecules is a hot model in many research fields. Herein, we report eco-friendly and facile technology to synthesize Ag-Au bimetallic nanocomposites. The synthesized nanocomposites are characterized by ultraviolet-visible spectroscopy, Fourier-transform infrared spectroscopy, X-ray diffraction, field emission scanning electron microscopy and high-resolution transmission electron microscopy. The synthesized nanocomposite can efficiently degrade Congo red and 4-nitrophenol in water and in the presence of sodium borohydride. The results show that it degrades Congo red and 4-nitrophenol entirely within 6 and $7 \mathrm{~min}$, respectively. These results could be useful for the green synthesis of Ag-Au bimetallic nanocomposites and help to remove organic dye molecules and nitroaromatics from wastewater.
\end{abstract}

Keywords: 4-nitrophenol; Congo red; azo dye; hydrogenation; degradation; wastewater treatment

\section{Introduction}

Aromatic compounds that contain a benzene ring, one or more hydroxyl group(s), one or more nitro group(s) and one or more azo bond(s) are toxic to the environment [1]. Nitrophenol compounds have broad industrial applications in dye, herbicide, pesticides, drugs and explosives [2]. One of the toxic, polluting agents is 4-nitrophenol, which causes headaches, drowsiness, stomach pain, chest pain and vomiting and damages organisms [3]. Furthermore, the dye compound contains a benzene ring and azo bonds and is used in textile industries, dyeing cotton, rubber, paper, paint and many other products [4]. The majority of dye molecules are harmful to human health and marine organisms, even at low concentrations [5]. Allergic dermatitis, skin allergy and dysfunction of the sex organs, kidney, brain, liver, etc., are caused by toxic dye molecules [6-8]. Moreover, wastewater containing colored substances prevents the penetration of oxygen and sunlight, which are needed for the survival of aquatic forms [9]. Congo red (1-naphthalene sulfonic acid, 3,3-(4,4-biphenylenebis(azo) bis(4-aminodisodium) salt) is a toxic anionic azo dye [4]. The complex molecular structures of Congo red dye inhibit degradation [10]. Thus, industrial wastewater containing 4-nitrophenol and dye molecules must be treated before discharge into a river or seawater. In the treatment of wastewater effluents, different techniques have been applied that are inexpensive, effective and eco-friendly for the detoxification of synthetic dyes and 4-nitrophenol [11-17]. Among the various treatment technologies, catalytic hydrogenation in the presence of sodium borohydride has been intensively investigated $[18,19]$. This model of catalytic hydrogenation reaction has been used widely due to the simplicity, cost-effectiveness and lower toxicity of $\mathrm{NaBH}_{4}$ and because it is easily monitored by $\mathrm{UV}$-Vis spectroscopy $[16,20]$. Moreover, the conversion of 4-nitrophenol to 4-aminophenol has great profit-making potential because 4-aminophenol is used as an important interme- 
diate for the synthesis of the antipyretic drug paracetamol [21]. The azo bonds in azo dye molecules can be cleaved easily using catalytic hydrogenation, and the dye molecules transform into less toxic and eco-friendly products. Different researchers have applied various catalytic systems for rapid hydrogenation of 4-nitrophenol and azo dye molecules in the last decade [20]. However, many of the surfactants and reactants used in these processes are toxic to human beings and the environment $[20,22]$. Thus, we should use green methods for the synthesis and application of metal nanoparticles [23]. Researchers have applied different flower extracts, leaf extracts and fruits to synthesize gold and silver nanoparticles in recent years $[16,19,24-27]$. The green synthesis of gold and silver nanoparticles using the most commonly available tea leaves extract is reported $[28,29]$. Tea leaves are among the most popular food and beverage ingredients with the highest flavonoid content, resulting in high antioxidant activity. The phytochemicals and amino acids found in tea leaves are responsible for reducing silver and gold ions [30,31]. Besides, the synthesis of bimetallic nanostructures and their catalytic activity are hot topics nowadays [27]. In recent years, Tripathy et al. reported on the synthesis of Ag-Au bimetallic nanocomposites using a biodegradable synthetic graft copolymer, hydroxyethyl starch-g-poly (acrylamide-coacrylic acid), as well as the study of their catalytic activities [32]. Bimetallic catalysts exhibit unique catalytic activities relative to their parent metals, which provide the ability to create new catalysts with better activity and selectivity [32,33]. The catalytic activity of metal nanoparticles relies significantly on their preparation methods, which control their size, shape and morphologies [32]. Green synthesis of bimetallic nanocomposites using green molecules would be more feasible and environmentally sustainable. Therefore, the synthesis of Ag-Au bimetallic nanocomposites using waste tea leaf extract is of interest in this paper. Furthermore, the synthesis of Ag-Au bimetallic nanoparticles/nanocomposites using waste tea leaf extract has not yet been reported.

Herein, we report an easy and facile synthesis approach of Ag-Au bimetallic nanocomposites using waste tea leaves extract to perform catalytic hydrogenation reaction of 4-nitrophenol (4-NP) and Congo red (CR) dye molecules in water.

\section{Materials and Methods}

\subsection{Materials}

Chloroauric acid tetrahydrate $\left(\mathrm{HAuCl}_{4} \cdot 4 \mathrm{H}_{2} \mathrm{O}\right)$, silver nitrate $\left(\mathrm{AgNO}_{3}\right)$, sodium borohydride $\left(\mathrm{NaBH}_{4}\right)$, 4-nitrophenol and Congo red dye were purchased from Sigma-Aldrich Chemicals Company, Seoul, Korea. Waste tea leaves were collected from India. Double-distilled water was used in all the experiments.

\subsection{Preparation of Waste Leaves Extract}

The collected tea leaves were washed with distilled water to remove dust particles if present. Then, $1.0 \mathrm{~g}$ of washed tea leaves was taken into a $250-\mathrm{mL}$ beaker with $100 \mathrm{~mL}$ of distilled water. The beaker was put on a hotplate with a magnetic stirrer at $80^{\circ} \mathrm{C}$ for $10 \mathrm{~min}$. Then, the beaker was kept at room temperature to settle down its contents. Following this, the tea color solution was filtered using Whatman 42 filter paper. The filtrate was collected in a sterile $100-\mathrm{mL}$ conical flask and was ready for immediate use or kept at $4{ }^{\circ} \mathrm{C}$ for later use.

\subsection{Synthesis of $A g-A u$ Nanocomposites}

The typical synthesis process for Ag-Au bimetallic nanocomposites (Ag-Au NCPs) was as follows: First, $40 \mathrm{~mL}$ of $10^{-3}(\mathrm{M})$ silver nitrate and $50 \mathrm{~mL}$ of $10^{-3}(\mathrm{M})$ chloroauric acid tetrahydrate were mixed in a $250-\mathrm{mL}$ beaker. Then, $3.0 \mathrm{~mL}$ of $50 \%$ diluted tea extract from the stock solution was added into the beaker by drops at room temperature. The immediate color change of the solution was observed and its intensity increased significantly over time and finally changed to reddish brown, as shown in Figure 1. The entire reaction process was carried out in the absence of sunlight to avoid any parent solution decomposition or nanocomposites. Silver nanocomposites (Ag NCPs) and gold nanocomposites (Au NPCPs) 
were prepared separately under the same experimental conditions to compare the color difference with $\mathrm{Ag}-\mathrm{Au} \mathrm{NCPs}$. The schematic representation of the synthesis process and catalytic activities of Ag-Au NCPs is shown in Figure 1.

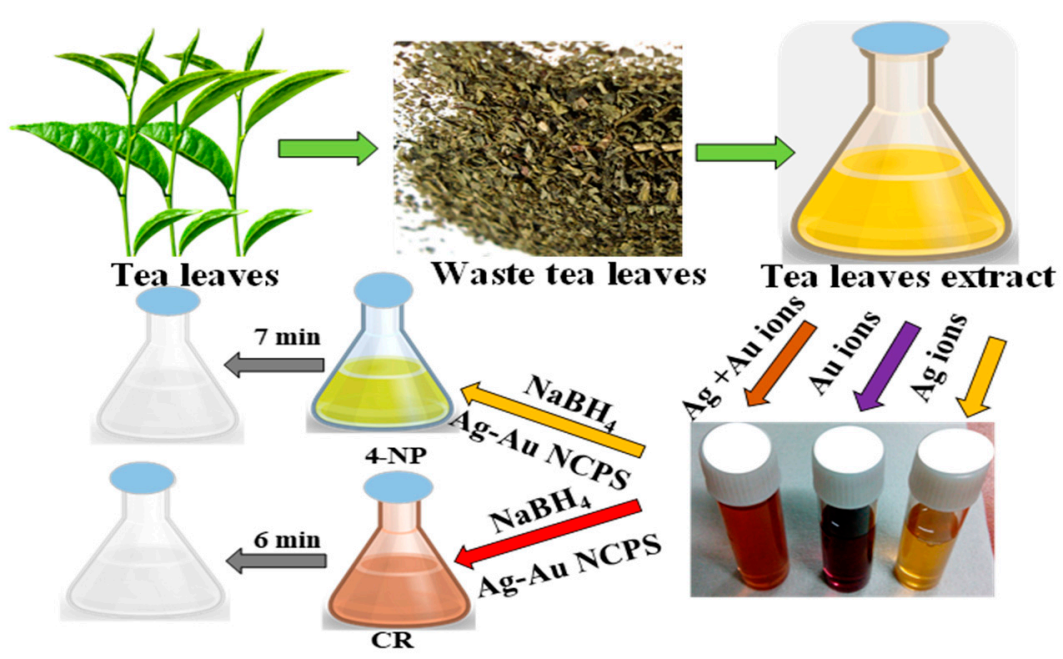

Figure 1. Schematic representation of the synthesis process and catalytic activity of Ag-Au nanocomposites (NCPs).

\subsection{Reduction of 4-Nitrophenol and Congo Red Azo Dye}

The reduction of 4-NP to 4-AP was carried out in a UV quartz cuvette with a length of $1 \mathrm{~cm}$ and a volume of $2.5 \mathrm{~mL}$. The cuvette took $1.5 \mathrm{~mL}$ of $10^{-4}(\mathrm{M}) 4-\mathrm{NP}$ water solution, and $20 \mu \mathrm{L} \mathrm{NaBH}_{4}$ methanol solution (prepared with $2 \mathrm{mg}$ 4-NP and $5 \mathrm{~mL}$ of distilled methanol) was added. After that, $20 \mu \mathrm{L}$ of Ag-Au NCPs hydrosol was mixed into the mixture and the catalytic activity was studied by kinetic analysis using UV-Vis spectroscopy. The spectrum was measured at an interval of $1 \mathrm{~min}$ at a wavelength of $600-200 \mathrm{~nm}$. Furthermore, the degradation of $1.5 \mathrm{~mL}$ of $10^{-4}(\mathrm{M})$ Congo red solution was followed by a similar procedure and the spectrum was taken at a wavelength of $800-200 \mathrm{~nm}$ at an interval of $1 \mathrm{~min}$.

\subsection{Material Characterization}

For optical, morphological and structural analyses, the synthesized nanocomposites were characterized by different characterization techniques. Colloidal samples were used for optical properties' investigations by UV-Vis spectroscopy (Shimadzu 1800, Shimadzu, Tokyo, Japan) and absorbance spectra were measured in a 1-cm quartz cuvette with a wavelength of 800-200 nm. A Perkin Elmer (Spectrum Two, Beaconsfield, UK) spectrometer was used to measure FT-IR spectra using $\mathrm{KBr}$ pellets in wavelengths from 4000 to $400 \mathrm{~cm}^{-1}$ to investigate changes in chemical functionality. Ag NCPs, Au NCPs and Ag-Au NCPs were first centrifuged, and then, the solid residue was dried in a vacuum oven at $60^{\circ} \mathrm{C}$. For the structural investigation, an X-ray diffractometer (Miniflex, Rigaku, Japan) was used to obtain the spectrum of bimetallic nanocomposites using $\mathrm{Cu} \mathrm{K} \alpha$ radiation at a scan rate of $1^{\circ} \mathrm{min}^{-1}$ in the range of $5-80^{\circ}$. The thick film of Ag-Au NCPs was produced by drying the colloidal sample onto a clean glass substrate for XRD measurements. Field emission scanning electron microscopy (FESEM, Gemini 500, Carl Zeiss) and high-resolution transmission electron microscopy (HR-TEM, JEM-2200 FS, JEOL Ltd., Japan) at $200 \mathrm{kV}$ were used for morphological investigation. Again, the drop-casting technique was used to obtain sufficient material for SEM analysis on glass substrates and HR-TEM analysis, using a lacey F/C 300 mesh Cu grid. 


\section{Results and Discussion}

A color change was observed with the formation of Ag-Au NCPs from yellowish to reddish brown. The distinct color change of the colloidal solution of the Ag-Au NCPs compared to the Ag NCPs and Au NCPs indicates the formation of the Ag-Au NCPs. The UV-Vis spectra of nanocomposites' hydrosols are shown in Figure 2.

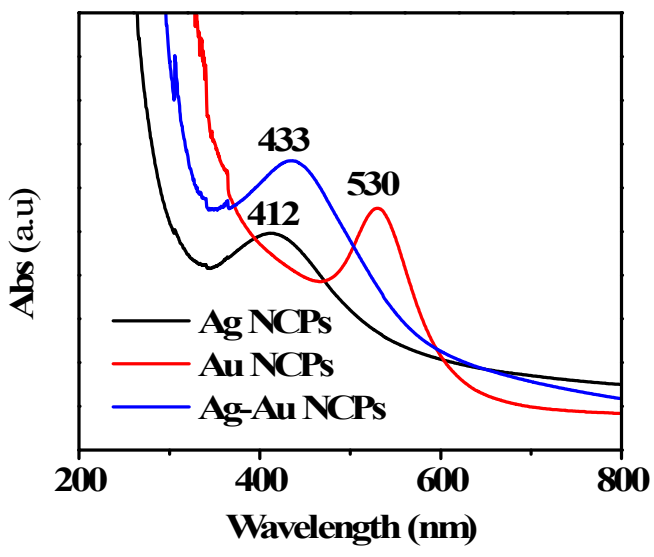

Figure 2. UV-Vis spectra of Ag NCPs, Au NCPs and Ag-Au NCPs' hydrosol.

The absorbance peak at $412 \mathrm{~nm}$ of the Ag NCPs and the peak at $530 \mathrm{~nm}$ of the Au NCPs suggest the formation of silver and gold nanocomposites. The well-defined peak at $433 \mathrm{~nm}$ is due to Ag-Au bimetallic nanocomposites, which is different from the peak positions of Ag and Au NCPs. By simple mixing of the Ag NCPs and Au NCPs, the SPR band at $433 \mathrm{~nm}$ was not obtained. Therefore, this result indicates that the absorbance band at $433 \mathrm{~nm}$ is due to the SPR band of Ag-Au bimetallic nanocomposites.

FT-IR spectra of waste tea samples and synthesized metal nanocomposites are shown in Figure 3 and the peak details are shown in Table 1.

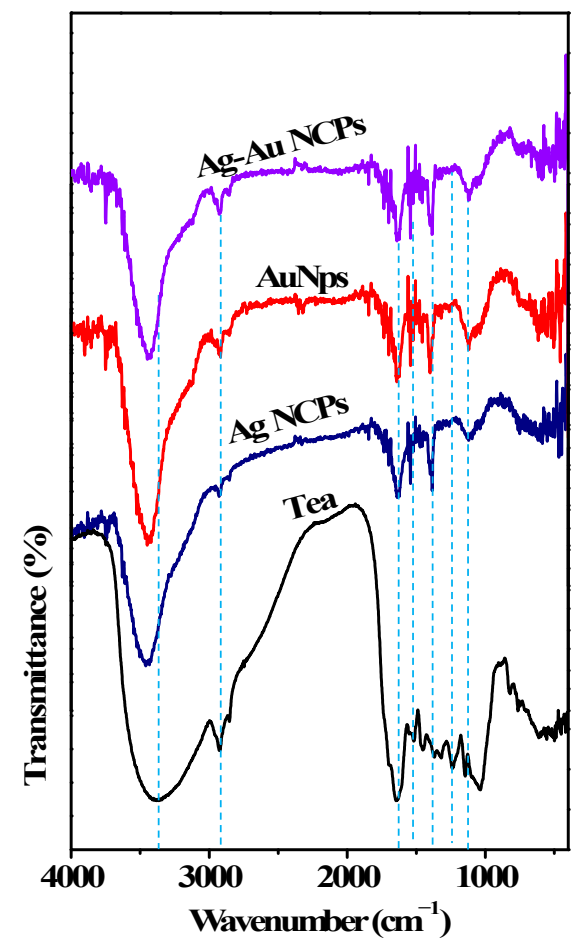

Figure 3. FT-IR spectra of tea leaves, Ag NCPs, Au NCPs and Ag-Au NCPs. 
Table 1. The FT-IR peak details of tea and monometallic and bimetallic nanocomposites.

\begin{tabular}{|c|c|c|c|c|c|}
\hline \multirow{2}{*}{ S1 No } & \multicolumn{4}{|c|}{ IR Peak at $\left(\mathrm{cm}^{-1}\right)$} & \multirow{2}{*}{ Assignment } \\
\hline & Tea & Ag NCPs & Au NCPs & Ag-Au NCPs & \\
\hline 1. & 3380 & 3459 & 3442 & 3428 & -OH stretching \\
\hline 2. & 2926 & 2928 & 2918 & 2928 & $-\mathrm{CH}_{2}$ asymmetric stretching \\
\hline 3. & 1637 & 1637 & 1637 & 1637 & $\mathrm{C}=\mathrm{O}$ stretching vibration \\
\hline 4. & 1454 & 1543 & 1543 & 1543 & $-\mathrm{CH}_{2}$ bending, ring stretch, $\mathrm{C}=\mathrm{C}, \mathrm{C}-\mathrm{O}$ \\
\hline 5. & 1238 & 1385 & 1396 & 1385 & $-\mathrm{C}(\mathrm{O})-\mathrm{O}$ stretching vibration and $-\mathrm{OH}$ in plane vibrations \\
\hline 6. & 1149 & 1116 & 1116 & 1116 & $-\mathrm{C}-\mathrm{O}$ stretching of $2^{\circ}$ alcohols \\
\hline 7. & 1043 & $\ldots$ & $\ldots$ & $\ldots$ & -C-O-C, anhydride, stretching \\
\hline
\end{tabular}

FT-IR spectrum of the tea sample shows a distinct difference from that of Ag-Au NCPs, Ag NCPs and Au NCPs.

The peak values of different functionalities are shifted to various frequencies. The peak at $1454 \mathrm{~cm}^{-1}$ is shifted to $1543 \mathrm{~cm}^{-1}$, the peak at $1238 \mathrm{~cm}^{-1}$ to $1385 \mathrm{~cm}^{-1}$ and the peak at $1149 \mathrm{~cm}^{-1}$ to $1116 \mathrm{~cm}^{-1}$ with increasing intensity due to the formation of nanocomposites with amide and $-\mathrm{OH}$ functionalities. Some more peaks also appeared that suggest the formation and stabilization of monometallic and bimetallic nanocomposites by waste tea leaves extract.

Figure 4 shows the wide-angle XRD patterns of Ag-Au nanocomposites. The diffractogram revealed the existence of distinct characteristic Ag-Au bimetallic peaks and cellulose peaks. The intense peak of Ag-Au NCPs is obvious at $2 \theta$ angles $38.2^{\circ}, 44.2^{\circ}, 64.4^{\circ}$ and $77.1^{\circ}$, most of which confirm the findings of previous works [34-36]. Others amorphous and crystalline peaks suggest cellulose crystallinity of tea leaves [37]. This result supports the formation of $\mathrm{Ag}$-Au bimetallic nanocomposites by waste tea leaves extract.

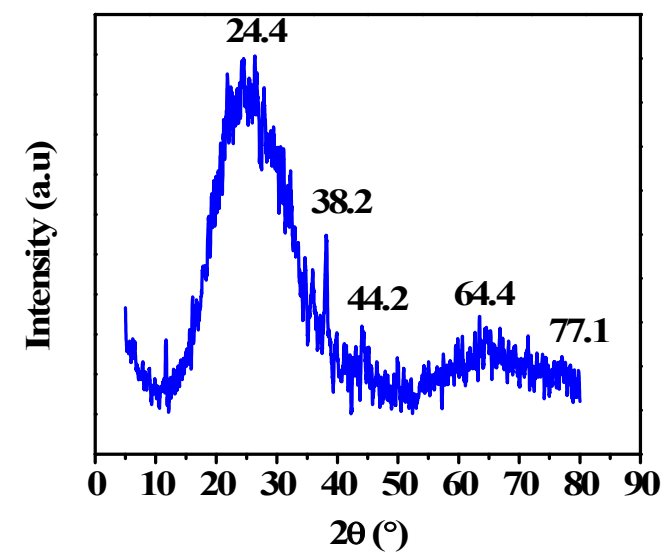

Figure 4. XRD pattern of Ag-Au bimetallic nanocomposites by tea leaves extract.

FESEM and HR-TEM were used to recognize the surface morphology and size of $\mathrm{Ag}-\mathrm{Au}$ NCPs, and EDS analysis was carried out to know the atomic percentage of Ag and $\mathrm{Au}$ in the bimetallic nanocomposites as shown in Figure 5. FESEM and HR-TEM micrographs (Figure 5a,b,d) show the spherical shape of Ag-Au nanoparticles in capping with shades in prickly pear (Opuntia). 

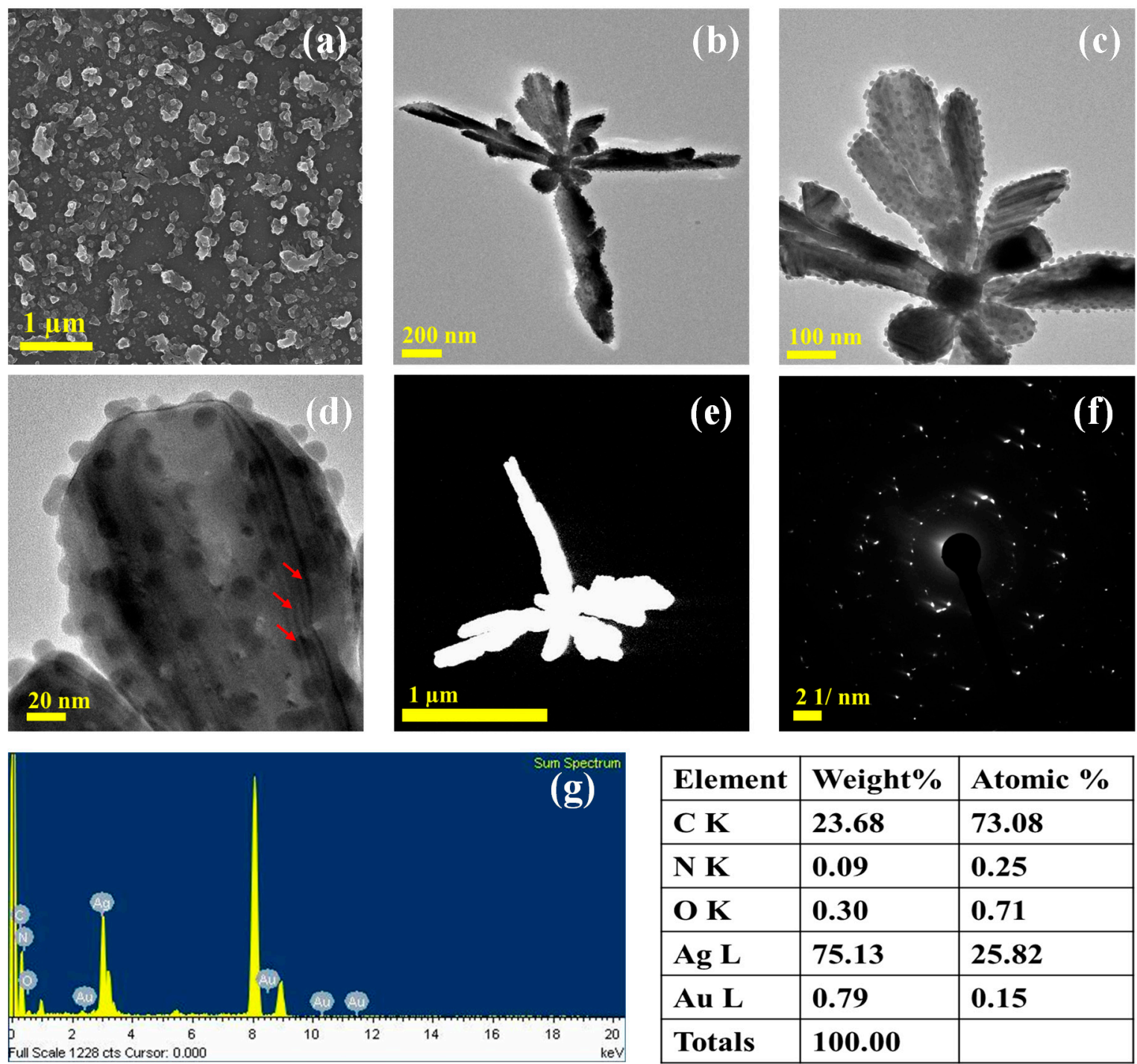

\begin{tabular}{|l|l|l|}
\hline Element & Weight\% & Atomic \% \\
\hline C K & 23.68 & 73.08 \\
\hline N K & 0.09 & 0.25 \\
\hline O K & 0.30 & 0.71 \\
\hline Ag L & 75.13 & 25.82 \\
\hline Au L & 0.79 & 0.15 \\
\hline Totals & 100.00 & \\
\hline
\end{tabular}

Figure 5. Micrographs of surface morphological studies: (a) FESEM, (b) TEM at $200 \mathrm{~nm}$, (c) TEM at $100 \mathrm{~nm}$, (d) high-resolution transmission electron microscopy (HR-TEM) at $20 \mathrm{~nm}$, red arrow highlights lattice fringes (e) electron image of selected area electron diffraction (SAED), (f) SAED pattern and (g) EDS spectrum and atomic percentage of elements of Ag-Au NCPs with tea leaves extract.

The shades on the nanocomposites' surface indicate the secondary materials, which are the phytochemicals of tea leaf extract [28,38]. It should be noted that these phytochemicals (mostly carboxylic acids) can efficiently reduce silver and gold to nanoparticles and act as a suitable capping agent, thus preventing them from aggregating $[28,38]$. The selected area electron diffraction (SAED) pattern (Figure 5f) displays that the Ag-Au nanocomposites are crystalline, and the crystalline ring represents the different lattice arrangement (Figure 5e). The EDS spectrum represents the Ag and Au's presence in Ag-Au NCPs at 2-3 and 8-12 keV, respectively. Bimetallic nanocomposites contain a higher amount of $\mathrm{Ag}$ than the $\mathrm{Au}$ content. The higher content of Ag in bimetallic nanocomposites was explained by the fact that $\mathrm{Ag}(\mathrm{I})$ ions attract more carboxylate groups than $\mathrm{AuCl}_{4}{ }^{-}$ions due to the smaller size of $\mathrm{Ag}(\mathrm{I})$ ions over larger $\mathrm{AuCl}_{4}{ }^{-}$ions [32].

The degradation in the presence of Ag-Au NCPs and $\mathrm{NaBH}_{4}$ of the Congo red dye molecules is shown in Figure 6a. Usually, the presence of azo bonds in the dye molecules is not reduced by $\mathrm{NaBH}_{4}$, but there is a reduction in metal nanoparticles' presence. The UV-Vis spectra of the Congo red dye's degradation display the appearance of two absorbance bands at 490 and $342 \mathrm{~nm}$ due to the $\mathrm{n} \rightarrow \pi^{*}$ and $\pi \rightarrow \pi^{*}$ electron transitions associated with 
azo bonds $(-\mathrm{N}=\mathrm{N}-)$. It is apparent from the figure that the intensity of both absorption peaks significantly decreases over time, and the color of the Congo red solution becomes colorless after $6 \mathrm{~min}$.
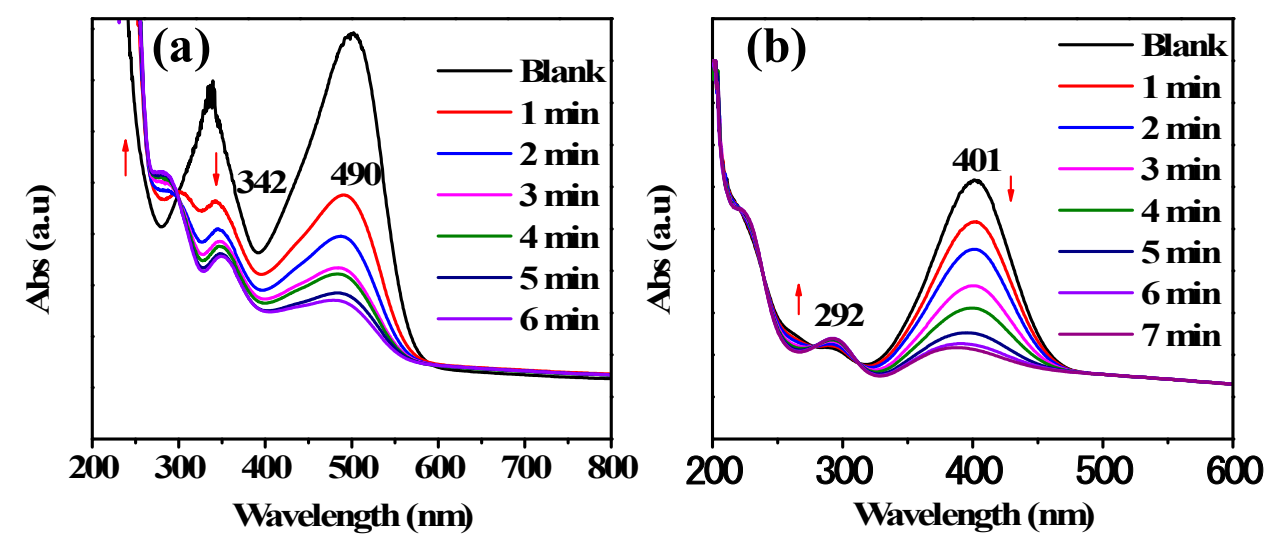

Figure 6. (a) UV-Vis spectra of Congo red degradation and (b) UV-Vis spectra of the reduction of 4NP to 4-AP after mixing with $\mathrm{NaBH}_{4}$ and Ag-Au NCPs (red arrows highlights changes in peak intensity).

The UV-Vis spectra of the reduction of 4-NP to 4-AP by Ag-Au NCPs in the presence of $\mathrm{NaBH}_{4}$ are shown in Figure 6b. The sharp absorbance peak at $401 \mathrm{~nm}$ gradually decreases over time due to the reduction of 4-NP. The new absorbance peak at $292 \mathrm{~nm}$ rises simultaneously due to the formation of 4-AP, and the reaction is completed in $7 \mathrm{~min}$. Rapid degradation of Congo red dye molecules and 4-NP occurs due to the greater surface area and irregular facets of the Ag-Au bimetallic NCPs. The mechanism of the azo bond cleavage and the reduction of $4-\mathrm{NP}$ by Ag-Au NCPs in the presence of $\mathrm{NaBH}_{4}$ have been explained in detail in our previous articles $[16,32]$ as well as in Figure 7. Besides, the reusability of bimetallic Ag-Au nanocomposites in the hydrogenation reaction of Congo red and 4-nitrophenol was studied based on previous studies [32]. The catalyst obtained in this study was used in the hydrogenation reactions, and after four cycles, it still displayed significant activity. These results indicate that the green synthesis of $\mathrm{Ag}-\mathrm{Au}$ bimetallic nanocomposites provides greater stability and catalytic activity.

(a)

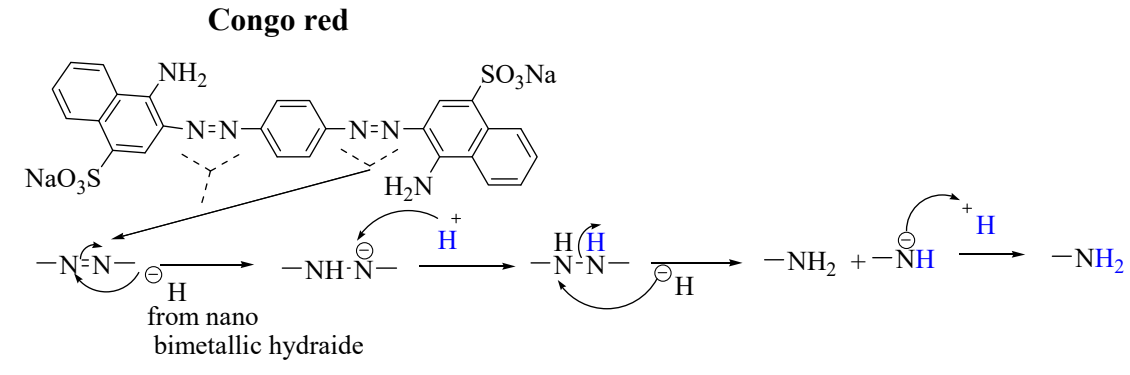

(b) $\mathrm{HO}-\mathrm{NO}_{2}$

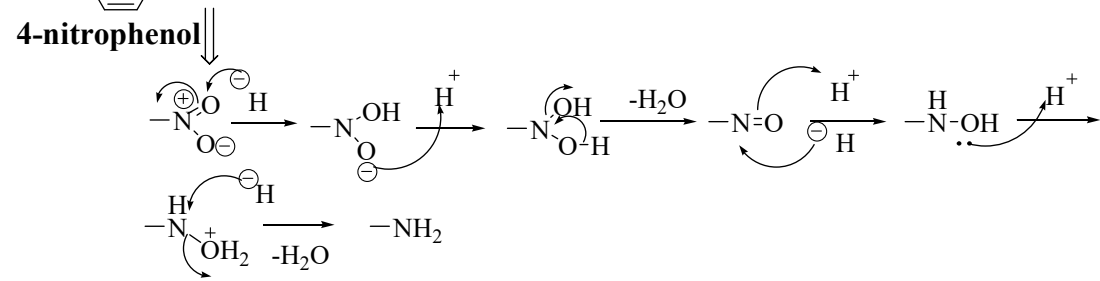

Figure 7. The probable mechanism of degradation of (a) azo bonds in Congo red and (b) nitro group in 4-nitrophenol in the presence of Ag-Au NCPs and $\mathrm{NaBH}_{4}$. 


\section{Conclusions}

Ag-Au bimetallic nanocomposites' green synthesis was carried out by a facile method using waste tea leaves extract in the water medium. The phytochemicals of the tea leaves extract act as a reducing and stabilizing agent. Ag-Au nanocomposites' formation was described based on extensive characterization results such as UV-Vis, FT-IR, XRD, FESEM, HR-TEM and EDS. The synthesized Ag-Au NCPs were used to study their performance as a bimetallic nanocatalyst in the degradation of Congo red dye molecules and 4-nitrophenol in the presence of $\mathrm{NaBH}_{4}$. The complete reduction in azo bonds and nitro groups occurred within 6 and $7 \mathrm{~min}$, respectively. The easy synthesis method and high catalytic activity of bimetallic Ag-Au nanocomposites could be useful for the removal of dyes and nitroaromatics from industrial effluent/wastewater purification.

Author Contributions: Formal analysis, project administration, funding acquisition, writing - review and editing: C.-W.K. Conceptualization, formal analysis, investigations, writing-original draft preparation, review and editing: H.K. Both authors have read and agreed to the published version of the manuscript.

Funding: This research was supported by the Basic Science Research Program through the National Research Foundation (NRF) of Korea funded by the Ministry of Education (NRF-2019R111A3A02059471) and was supported under the framework of the International Cooperation program managed by the NRF of Korea (NRF-2020K2A9A2A08000181).

Institutional Review Board Statement: Not applicable.

Informed Consent Statement: Not applicable.

Data Availability Statement: Not applicable.

Conflicts of Interest: The authors declare that they have no known competing financial interests or personal relationships that could have appeared to influence the work reported in this paper.

\section{References}

1. Jing, Z; Dai, X.; Xian, X.; Zhang, Q.; Zhong, H.; Li, Y. Novel Ternary heterogeneous reduction graphene oxide (RGO) $/ \mathrm{BiOCl} / \mathrm{TiO}_{2}$ nanocomposites for enhanced adsorption and visible-light induced photocatalytic activity toward organic contaminants. Materials 2020, 13, 2529. [CrossRef]

2. Ju, K.S.; Parales, R.E. Nitroaromatic compounds, from synthesis to biodegradation, Microbiol. Mol. Biol. Rev. 2010, 74, $250-272$. [CrossRef] [PubMed]

3. Wang, N.; Lv, G.; He, L.; Sun, X. New insight into photodegradation mechanisms, kinetics and health effects of p-nitrophenol by ozonation in polluted water. J. Hazard. Mater. 2021, 403, 123805. [CrossRef] [PubMed]

4. Yang, Y.; Ali, N.; Khan, A.; Khan, S.; Khan, S.; Khan, H.; Xiaoqi, S.; Ahmad, W.; Uddin, S.; Ali, N.; et al. Chitosan-capped ternary metal selenide nanocatalysts for efficient degradation of Congo red dye in sunlight irradiation. Int. J. Biol. Macromol. 2021, 167, 169-181. [CrossRef]

5. Salleh, M.A.M.; Mahmoud, D.K.; Karim, W.A.W.A.; Idris, A. Cationic and anionic dye adsorption by agricultural solid wastes: A comprehensive review. Desalination 2011, 280, 1-13. [CrossRef]

6. Brown, M.A.; de Vito, S.C. Predicting azo dye toxicity. Crit. Rev. Environ. Sci. Technol. 1993, 23, 249-324. [CrossRef]

7. Argumedo-Delira, R.; Gómez-Martínez, M.J.; Uribe-Kaffure, R. Trichoderma biomass as an alternative for removal of congo red and malachite green industrial dyes. Appl. Sci. 2021, 11, 448. [CrossRef]

8. He, W.; Zhang, Y.; Fatehi, P. Sulfomethylated kraft lignin as a flocculant for cationic dye. Colloids Surf. Physicochem. Eng. Asp. 2016, 503, 19-27. [CrossRef]

9. Culp, S.J.; Beland, F.A. Malachite green: A toxicological review. J. Am. Coll. Toxicol. 1996, 15, 219-238. [CrossRef]

10. Aziz, A.; Ali, N.; Khan, A.; Bilal, M.; Malik, S.; Ali, N.; Khan, H. Chitosan-zinc sulfide nanoparticles, characterization and their photocatalytic degradation efficiency for azo dyes. Int. J. Biol. Macromol. 2020, 153, 502-512. [CrossRef] [PubMed]

11. Wekoye, J.N.; Wanyonyi, W.C.; Wangila, P.T.; Tonui, M.K. Kinetic and equilibrium studies of Congo red dye adsorption on cabbage waste powder. Environ. Chem. Ecotoxicol. 2020, 2, 24-31. [CrossRef]

12. Zhu, J.; Li, J.; Li, Y.; Guo, J.; Yu, X.; Peng, L.; Han, B.; Zhu, Y.; Zhang, Y. Adsorption of phosphate and photodegradation of cationic dyes with BiOI in phosphate-cationic dye binary system. Sep. Purif. Technol. 2019, 223, 196-202. [CrossRef]

13. Nadeem, K.; Guyer, G.T.; Keskinler, B.; Dizge, N. Investigation of segregated wastewater streams reusability with membrane process for textile industry. J. Clean. Prod. 2019, 228, 1437-1445. [CrossRef]

14. Chen, S.; Du, J.; Shen, L.; Chen, W. In situ decoration of Ag nanoparticles on hierarchical TiO2 nanowire nanofibers with enhanced catalytic activity used for the removal of 4-nitrophenol. J. Phys. Chem. Solids. 2021, 149, 109784. [CrossRef] 
15. Sasmal, D.; Maity, J.; Kolya, H.; Tripathy, T. Study of congo red dye removal from its aqueous solution using sulfated acrylamide and N, N- dimethyl acrylamide grafted amylopectin. J. Water Process Eng. 2017, 18, 7-19. [CrossRef]

16. Kolya, H.; Kuila, T.; Kim, N.H.; Lee, J.H. Bioinspired silver nanoparticles/reduced graphene oxide nanocomposites for catalytic reduction of 4-nitrophenol, organic dyes and act as energy storage electrode material. Compos. Part B Eng. 2019, $173,106924$. [CrossRef]

17. Kolya, H.; Tripathy, T. Hydroxyethyl Starch-g-Poly-(N,N-dimethylacrylamide-co-acrylic acid): An efficient dye removing agent. Eur. Polym. J. 2013, 49, 4265-4275. [CrossRef]

18. Cyganowski, P. Fully recyclable gold-based nanocomposite catalysts with enhanced reusability for catalytic hydrogenation of p-nitrophenol. Colloids Surf. Physicochem. Eng. Asp. 2021, 612, 125995. [CrossRef]

19. Kolya, H.; Maiti, P.; Pandey, A.; Tripathy, T. Green synthesis of silver nanoparticles with antimicrobial and azo dye (Congo red) degradation properties using Amaranthus gangeticus Linn leaf extract. J. Anal. Sci. Technol. 2015, 6, 33. [CrossRef]

20. Naseem, K.; Farooqi, Z.H.; Begum, R.; Irfan, A. Removal of Congo red dye from aqueous medium by its catalytic reduction using sodium borohydride in the presence of various inorganic nano-catalysts: A review. J. Clean. Prod. 2018, 187, 296-307. [CrossRef]

21. Kong, X.; Sun, Z.; Chen, M.; Chen, C.; Chen, Q. Metal-free catalytic reduction of 4-nitrophenol to 4-aminophenol by N-doped graphene. Energy Environ. Sci. 2013, 6, 3260-3266. [CrossRef]

22. Zhao, P.; Feng, X.; Huang, D.; Yang, G.; Astruc, D. Basic concepts and recent advances in nitrophenol reduction by gold- and other transition metal nanoparticles. Coord. Chem. Rev. 2015, 287, 114-136. [CrossRef]

23. Shamaila, S.; Sajjad, A.K.L.; Ryma, N.-A.; Farooqi, S.A.; Jabeen, N.; Majeed, S.; Farooq, I. Advancements in nanoparticle fabrication by hazard free eco-friendly green routes. Appl. Mater. Today. 2016, 5, 150-199. [CrossRef]

24. Ghosh, S.; Patil, S.; Ahire, M.; Kitture, R.; Gurav, D.D.; Jabgunde, A.M.; Kale, S.; Pardesi, K.; Shinde, V.; Bellare, J.; et al. Gnidia tglauca flower extract mediated synthesis of gold nanoparticles and evaluation of its chemocatalytic potential. J. Nanobiotechnol. 2012, 10, 17. [CrossRef]

25. Nahar, K.N.; Rahaman, M.H.; Khan, G.M.A.; Islam, M.K.; Al-Reza, S.M. Green synthesis of silver nanoparticles from Citrus sinensis peel extract and its antibacterial potential. Asian J. Green Chem. 2021, 5, 135-150. [CrossRef]

26. Sundarrajan, S.K.; Pottail, L. Green synthesis of bimetallic Ag@Au nanoparticles with aqueous fruit latex extract of Artocarpus heterophyllus and their synergistic medicinal efficacies. Appl. Nanosci. 2021, 11, 971-981. [CrossRef]

27. Deng, S.; Zhao, B.; Xing, Y.; Shi, Y.; Fu, Y.; Liu, Z. Green synthesis of proanthocyanidins-functionalized Au/Ag bimetallic nanoparticles. Green Chem. Lett. Rev. 2021, 14, 43-48. [CrossRef]

28. Chandra, A.; Bhattarai, A.; Yadav, A.K.; Adhikari, J.; Singh, M.; Giri, B. Green synthesis of silver nanoparticles using tea leaves from three different elevations. ChemistrySelect 2020, 5, 4239-4246. [CrossRef]

29. Begum, N.A.; Mondal, S.; Basu, S.; Laskar, R.A.; Mandal, D. Biogenic synthesis of Au and Ag nanoparticles using aqueous solutions of Black Tea leaf extracts. Colloids Surf. B Biointerfaces 2009, 71, 113-118. [CrossRef] [PubMed]

30. Tounekti, T.; Joubert, E.; Hernández, I.; Munné-Bosch, S. Improving the polyphenol content of tea, CRC. Crit. Rev. Plant Sci. 2013, 32, 192-215. [CrossRef]

31. Onitsuka, S.; Hamada, T.; Okamura, H. Preparation of antimicrobial gold and silver nanoparticles from tea leaf extracts. Colloids Surf. B. Biointerfaces 2019, 173, 242-248. [CrossRef]

32. Tripathy, T.; Kolya, H.; Jana, S.; Senapati, M. Green synthesis of Ag-Au bimetallic nanocomposites using a biodegradable synthetic graft copolymer; hydroxyethyl starch-g-poly (acrylamide-co-acrylic acid) and evaluation of their catalytic activities. Eur. Polym. J. 2017, 87, 113-123. [CrossRef]

33. Zhang, Y.; Li, G.; Hu, Y. Fabrication of bimetallic nanoparticles modified hollow nanoporous carbons derived from covalent organic framework for efficient degradation of 2,4-dichlorophenol. Chin. Chem. Lett. 2021, in press. [CrossRef]

34. Berahim, N.; Basirun, W.J.; Leo, B.F.; Johan, M.R. Synthesis of Bimetallic Gold-Silver (Au-Ag) Nanoparticles for the Catalytic Reduction of 4-Nitrophenol to 4-Aminophenol. Catalyst 2018, 8, 412. [CrossRef]

35. Diem, P.N.H.; Phuong, T.N.M.; Hien, N.Q.; Quang, D.T.; Hoa, T.T.; Cuong, N.D. Silver, gold, and silver-gold bimetallic nanoparticle-decorated dextran: Facile synthesis and versatile tunability on the antimicrobial activity. J. Nanomater. 2020, 2020, 7195048. [CrossRef]

36. Elemike, E.E.; Onwudiwe, D.C.; Fayemi, O.E.; Botha, T.L. Green synthesis and electrochemistry of Ag, Au, and Ag-Au bimetallic nanoparticles using golden rod (Solidago canadensis) leaf extract. Appl. Phys. A. 2019, 125, 42. [CrossRef]

37. Arie, A.A.; Tekin, B.; Demir, E.; Demir-Cakan, R. Utilization of The Indonesian's spent tea leaves as promising porous hard carbon precursors for anode materials in sodium ion batteries. Waste Biomass Valoris. 2020, 11, 3121-3131. [CrossRef]

38. Benakashani, F.; Allafchian, A.R.; Jalali, S.A.H. Biosynthesis of silver nanoparticles using Capparis spinosa L. leaf extract and their antibacterial activity. Karbala Int. J. Mod. Sci. 2016, 2, 251-258. [CrossRef] 